\title{
Les termes basés sur coach et coaching en polonais
}

\begin{abstract}
Résumé
Compte tenu de l'apparition ces dernières années en polonais d'une vague de termes construits autour du noyau constitué par les mots coach et coaching, nous nous proposons dans notre article de rendre compte de ce phénomène intéressant. Seront prises en considération les adaptations au système grammatical et la circulation en polonais de ces néologismes, collectés dans différents types de documents, traditionnels et publiés dans Internet.
\end{abstract}

Mots-clés : lexicologie, néologisme, emprunt, adaptations grammaticales

\section{Summary}

In Polish in recent years multiple terms built around words coach and coaching have appeared. The article analyses these neologisms, concentrating on their adaptations to grammatic system of Polish language as well as their appearance in multiple types of traditional documents and those published in the Internet.

Keywords: lexicology, neologism, borrowing, grammatical adaptations

\section{Introduction}

En décembre 2004, Le Figaro constatait dans l'article intitulé « La mode du "coaching" envahit tous les domaines " l'apparition d'un certain nombre de nouvelles professions centrées sur la sphère privée, mais basées en même temps sur le modèle de l'« executive coaching », connu dès les années 1990 dans le monde 
de l'entreprise et auparavant dans le sport. En effet, elles visent désormais différents domaines de la vie humaine dans lesquels l'intervention d'un coach peut être ressentie comme nécessaire, tels la santé, la beauté, l'éducation, voire l'amour et le sexe. Sur le plan lexical, ce foisonnement d'activités a pour résultat l'apparition en français de plusieurs termes nouveaux construits autour du noyau constitué par les mots coaching et coach, à commencer par life coaching, suivi, entre autres, de coach sportif, coach santé, coach éducation, coach love, coach relooking, coach culture ou coach déco.

En Pologne, le mot coach, au sens d'« entraîneur », a d'abord été utilisé dans le contexte sportif; cependant, avec l'implantation dans le pays de sociétés multinationales, il s'est répandu (accompagné aussi du terme coaching) dans le langage de leurs jeunes employés, puis aussi dans le discours public. Dans le présent article, nous nous proposons tout d'abord de rendre compte de l'apparition du mot coach (et de coaching) en polonais, avant de rendre compte de la présence de termes basés sur ces deux emprunts, que nous avons collectés dans internet entre le $1^{\mathrm{er}}$ mai et le $1^{\mathrm{er}}$ octobre 2016 (en nous limitant aux pages web publiées en polonais). Au fur et à mesure de leur adaptation au système lexical du polonais, ces mots ont subi des modifications formelles et sémantiques, ce dont nous rendrons compte dans la partie suivante de notre étude. Nous vérifierons aussi la présence des termes pris en considération dans les dictionnaires de la langue polonaise, traditionnels et en ligne, afin de pouvoir déterminer la façon dont ils sont intégrés dans cette langue.

\section{L'origine des mots coach et coaching et leur présence dans les dictionnaires français et polonais}

Une recherche diachronique montre que le français a emprunté le mot coach à l'anglais, mais que son histoire est en fait plus longue et plus compliquée. Selon l'Oxford dictionary, le mot vient du hongrois kocsi szekér « wagon de Kocs», éponyme créé sur la base du nom de la ville Kocs, siège d'un relais de poste sur la route de Vienne à Pest. Passé d'abord en français (d'après le DEF par l'intermédiaire de l' allemand) et assimilé sous la forme coche pour dénommer « une grande voiture, non suspendue, tirée par des chevaux, qui faisait le service des voyageurs ", ce qui est attesté déjà en 1545 (cf. Négociations de la France dans le Levant, ou Correspondances, mémoires et actes diplomatiques des ambassadeurs 
de France à Constantinople et des ambassadeurs, envoyés ou résidents à divers titres à Venise (...), publiés par E. Charrière), le substantif, encore au $16^{\mathrm{e}}$ siècle, a été emprunté par l'anglais avec la même signification (mais avec la modification de la forme en $(0 a c h$ ), en donnant lieu aussi à la création à sa base d'un verbe intransitif. Au $18^{\mathrm{e}} \mathrm{s}$. cependant, le mot a développé en anglais aussi un autre sens, celui de « répétiteur qui aide un étudiant en vue d'une épreuve (l'entraînant comme une voiture) ", puis, d'une manière plus spécifique, aussi "entraîneur sportif » et a servi de base à la dérivation d'un verbe transitif. Comme l'atteste le $T L F i$, c'est avec ces deux nouveaux sens - en indiquant leur emploi en français en 1965 (mais aussi avec les significations antérieures, à savoir " grande voiture fermée comportant deux portes latérales », datée de 1897, et « automobile dont la carrosserie à quatre glaces latérales ne comporte que deux portes ", datée de 1951) - que le mot est réemprunté en français. Ce même dictionnaire présente aussi, dans la même entrée, une remarque supplémentaire concernant l'existence de deux dérivés utilisés dans le vocabulaire du sport, à savoir le verbe coacher " entraîner » et le substantif coaching « entraînement ». Un sens spécifiquement français du mot coach semble être pourtant développé dans le discours du cinéma, où il désigne «le professeur qui guide un acteur étranger dans son français quelquefois hésitant ». Il est par contre à souligner que le TLFi ne rend pas du tout compte du sens nouveau (proche de " conseiller », signalé par l'article publié par Le Figaro en 2004, cité ci-dessus) ni ne prévoit d'entrée séparée pour le terme coaching. Il n'en est pas de même du Dictionnaire Larousse en ligne qui définit coach comme : "Entraîneur d'une équipe, d'un sportif de haut niveau » et qui ajoute une nouvelle définition : "Conseiller professionnel qui cherche à développer les performances d'un salarié, d'un acteur, d'un chef d'entreprise, etc. ». Ce même dictionnaire indique aussi le mot coaching, défini comme « fonction, activité de coach ». Le Wiktionnaire suit le même ordre dans la présentation des définitions relatives au mot coach, à commencer par le sens d'« entraîneur sportif », en énumérant ensuite celui d' ' entraîneur personnel » et en troisième lieu celui de " grande voiture fermée ». Le même dictionnaire signale aussi les dérivés coacher et coaching, ce deuxième avec, entre autres, en troisième position, le sens de «mentorat, accompagnement ou entraînement personnel, de conseil professionnel ou personnel; guidance (psychologique)».

Quant au polonais, les dictionnaires traditionnels de référence (Wielki Słownik Języka Polskiego et Słownik Języka Polskiego) notent l'existence du mot $k o c z$, désignant « une grande voiture pour le transport de plusieurs personnes, souvent couverte, à quatre roues, tirée par des chevaux ». Le dictionnaire étymologique de Karol Brückner indique son origine hongroise, en précisant cependant qu'il s'agit d'un mot existant dans plusieurs langues européennes, qui est 
probablement passé en polonais par l'intermédiaire d'une de ces langues, et n'est pas venu du hongrois directement (il évoque le français, l'allemand ou l'anglais). Le mot coach [kocz] à son tour, emprunté certainement dans les dernières décennies du $20^{\mathrm{e}} \mathrm{s}$. directement à l'anglais, est noté seulement par le dictionnaire $S J P$ qui en propose deux définitions, en indiquant dans les deux cas son usage dans un milieu spécifique : soit «trener, zwłaszcza drużyny piłkarskiej» 'entraîneur, surtout d'une équipe de football', soit « nadwozie samochodu osobowego » 'carrosserie d'une voiture'. Le sens nouveau du mot coach n'est pas du tout mentionné, par contre le dictionnaire contient l'entrée coaching [koczing] qu'il définit comme «aktywna współpraca szefa z pracownikiem w celu eliminowania błędów w pracy i wspierania rozwoju pracownika », 'coopération active du chef avec l'employé afin d'éliminer ses erreurs dans le travail et afin de favoriser son développement'. Le dictionnaire note aussi l'adjectif coachingowy.

Ce n'est que le dictionnaire en ligne Wikislownik qui indique le nouveau sens en première position, à savoir : « osobisty trener pomagający odkryć właściwą drogę do celu, używając własnych umiejętności, doświadczenia życiowego, różnorodnych technik i narzędzi ", 'entraîneur personnel aidant à découvrir comment arriver au but grâce à ses propres capacités et son expérience, en utilisant différentes techniques et instruments'.

Comme le montre cette étude de la présence des mots coach et coaching dans les dictionnaires, les significations liées à « entraînement personnel » sont dans les deux langues visiblement ressenties comme encore trop récentes pour figurer dans les dictionnaires traditionnels. Il est par contre significatif que les sens en question trouvent bien leur place et leurs définitions dans les dictionnaires en ligne comme Wikislownik et Wiktionnaire.

\section{Les adaptations des mots coach et coaching au système grammatical du polonais}

Les phonèmes dont se composent les mots anglais coach et coaching ne constituent pas une difficulté majeure pour être prononcés par un locuteur polonais ; cependant les deux mots ont subi une assimilation phonétique : [kəot $f]$ anglais est passé à [kəwt ] et [kəv.tfin] à [kəwtfing]. C'est donc d'abord la diphongue [əo] qui est remplacée par en [॰] accompagnée de la semi-voyelle [w], et dans coaching, d'une manière supplémentaire, la consonne [n] est remplacée par le groupe consonantique prononcé [ng]. 
En ce qui concerne la graphie, les deux mots apparaissent sous différentes formes, parmi lesquelles la forme originale anglaise, non modifiée, est de loin la plus fréquente. Cependant, des formes « polonisées » existent aussi. Pour coaching c'est tout d'abord koaching où, en accord avec l'orthographe polonaise, le son $[\mathrm{k}$ ] est représenté par le graphème $\mathrm{K}$. On note aussi la graphie kołczing où [w] est représenté par le graphème $€$, alors que [t $\mathrm{t}$ ] l'est par le graphème $\mathrm{CZ}$. Le mot coach apparaît à son tour aussi sous une forme vaguement polonisée koucz dans laquelle, à part l'introduction du graphème (k), c'est la modification de (oa) en (ou) qui est censé noter la prononciation de [w]. Dans la forme kołcz le phonème $[w]$ est aussi rendu au moyen du graphème polonais $€$.

Pour ce qui est de l'assimilation morphologique des deux mots, celle-ci doit être considérée comme particulièrement marquée. En premier lieu, notons que les deux mots, même sous leurs formes graphiques anglaises (qui reflètent le soin du locuteur de les prononcer «à l'anglaise »), ont subi une adaptation au système de la déclinaison et fonctionnent avec les terminaisons des cas polonais. Le tableau ci-dessous présente la déclinaison du mot coach en polonais au singulier et au pluriel:

\begin{tabular}{|c|c|c|}
\hline \multicolumn{1}{|c|}{ cas } & singulier & pluriel \\
\hline nominatif & coach & coachowie \\
\hline génitif & coacha & coachów \\
\hline datif & coachowi & coachom \\
\hline accusatif & coacha & coachów \\
\hline ablatif & coachem & coachami \\
\hline locatif & coachu & coachach \\
\hline vocatif & coachu & coachowie \\
\hline
\end{tabular}

La présence en ligne de ce tableau semble témoigner d'un côté du besoin que les locuteurs polonophones peuvent ressentir à utiliser des formes déclinées, mais en même temps de leur sentiment d'instabilité par rapport aux dites formes. Ce besoin découle sans doute du fait de l'absence en polonais de la terminaison nominale analogue : si $\left(\int\right)$, surtout dans la terminaison [a $\left.\int\right]$, apparaît bien comme la consonne finale de la catégorie de noms d'agent au masculin, il n'en est pas de même du groupe [owt $\int$, ce qui doit constituer une source d'hésitation quant à la forme à choisir.

Le même dictionnaire ne présente pas le même type de tableau pour coaching, mais de nombreux emplois de ce mot sous formes fléchies relevés dans notre corpus témoignent bien de son assimilation par le système des cas en polonais. L'exemple ci-dessous (1) montre notamment la forme coachingowi qui est un datif de coaching: 
1. Dzięki coachingowi zmieniają się liderzy, organizacje, może zmieniać się także nauczyciel i szkoła.

[Grâce au coaching des leaders, des organisations changent, il est possible aussi que changent l'enseignant et l'école.]

www.humanitas.edu.pl/APiB_Coaching_oswiata

Les deux mots ont aussi servi de base à de nombreuses dérivations en polonais. En premier lieu il convient de mentionner des tentatives de créer le féminin du substantif coach, ce qui semble poser des problèmes plutôt esthétiques que morphologiques, étant donné que des noms d'agent d'origine étrangère se soumettent assez régulièrement à ce procédé depuis longtemps (ex. : pasażer / pasażerka " passager / passagère »; fryzjer / fryzjerka " coiffeur / coiffeuse », etc.). Le problème est posé par les noms de postes ou de métiers à prestige (ex. minister « ministre ", premier " premier ministre »), dont les féminins proposés (ministra «femme ministre », premierka « femme premier ministre ) sont loin d'être admis par le système de la langue. Tout comme en français, en polonais les féminins de ces noms sont souvent perçus comme moins sérieux, voire « laids». C'est de là que vient probablement aussi l'hésitation des locuteurs, qui sont embarrassés par le choix du masculin pour désigner une femme coach ou par celui de la forme féminisée par l'adjonction du suffixe $-k a$, coach $k a$, ce dont rend compte l'exemple (2) :

2. Ela Soszyńska, Z zawodu hispanistka, Z pochodzenia góralka, Z pozoru zorganizowana, Od pewnego momentu certyfikowany coach (coachka?)

['Ela Soszyńska, hispaniste de profession, montagnarde d'origine, Bien organisée en apparence, coach (coachka?) certifiée depuis un certain temps']

www.wawelowe.pl

Notons que ce féminin ne fonctionne que d'une manière marginale (dans internet 990 résultats le 2.09.16) par rapport à celle de coach (393000 à la même date); de plus, ses emplois semblent toujours marqués comme plaisants et/ou familiers, comme c'est le cas dans l'exemple (3) :

3. Boska Coachka - tak pomyślałam o Grażynie, gdy zaczętyśmy pracować.

[Une coach divine - c'est ce que j'ai pensé de Grażyna, quand nous avons commencé à travailler ensemble]

coachingdobrychmysli.com 
L'instabilité de la forme en question est confirmée aussi par son orthographe hésitante :

- $\quad$ soit le morphème - $k a$ indiquant le féminin se trouve joint au mot de base coach ou kołcz, selon le modèle des formes originelles (autochtones) (4) :

4. (...) smutna coachka (kołczka-trenerka), Muza porzucona z wierszem na ręku (...) [(...) une coach triste (une coach-entraîneuse), une Muse abandonnée avec un poème dans ses bras (...)]

Mądre wygłupy - E-teatr.pl

- soit les morphème du féminin est semi-conjoint, à l'aide du trait d'union, coach-ka (5), ou de l'apostrophe coach'ka (6), graphies qui n'existent pas en polonais pour marquer le féminin.

5. Coach-ka mnie wkurzała, bo nie daje soba sterować, ale chce sterować mną. [Ma coach me rendait furieuse, car elle ne se laisse pas guider alors qu'elle veut me guider.]

krainatrolli.blox.pl/2013/01/Wyrodne-dziecko.html

6. Klaudia Pingot, Trenerka rozwój osobistego i coach'ka, autorka książek i wielu programów szkoleniowych (...).

[Klaudia Pingot, entraîneuse du développement personnel et coach, auteur de livres et de programmes d'enseignement (...)]

smart-coaching.pl/czlonek-zespolu/klaudia-pingot

Le mot coach a servi de base aussi à la création d'un verbe. Défini par le dictionnaire de l'OJUW comme "prowadzić coaching", " mener le coaching ", il apparaît avec deux graphies, coachować (3 030 résultats) et, moins fréquemment, kołczować (791 résultats). Si la première forme apparait surtout dans des contextes que l'on pourrait considérer comme standards, la deuxième relève essentiellement du jargon des jeunes employés des multinationales. Voici la définition (7) qu'en donne le dictionnaire en ligne (à vocation, entre autres, ludique) intitulé Słownik slangu polsko-angielskiego:

7. Kołczować to odbywać rozmowę z pracownikiem w celu poprawienia jego wydajności, kołczować oznacza tyle co trenować, motywować i rozwijać jednocześnie. 
[Coacher c'est avoir un entretien avec son employé en vue de son développement, coacher signifie la même chose qu'entraîner, motiver et développer en même temps.]

http://www.ponglish.org

Le verbe apparaît cependant aussi avec le sens lié à l'entraînement personnel dans différents domaines, ce qui semble constituer le transfert de ses emplois traditionnels en anglais. L'exemple ci-dessous montre le cas de l'emploi assez général du verbe en question, restreint cependant au domaine de la psychologie :

8. Podobny, moralny obowiązek ma coach względem klienta. Jeżeli stwierdzi, że nie powinien $z$ danego tematu kołczować - powinien odesłać do specjalisty.

[Un coach a un devoir moral envers son client. S'il constate qu'il ne peut pas le coacher dans un domaine, il doit diriger le client vers un spécialiste.]

http://www.goldenline.pl/(2008)

En accord avec le système verbal polonais dans lequel l'opposition entre l'aspect accompli et inaccompli est cruciale, le verbe kołczować, ayant un aspect inaccompli, a servi de base à la création de la forme préfixée au moyen du morphème s-, skołczować, exprimant un aspect accompli. Ce mot (avec 3780 résultats sur google.pl) est glosé par un autre dictionnaire en ligne du jargon de multinationales comme "nauczyć kogoś czegoś », « enseigner quelque chose à quelqu'un ».

9. Część klientów zetknęła się z coachingiem, na przykład w korporacji i słowo scoachować czy raczej skołczować często kojarzy im się z nieprzyjemna rozmowa oceniajaca anizeli z praca nad swoim rozwojem.

[Une partie des clients a eu un contact avec le coaching, par exemple dans une multinationale, et ils associent le mot scoachować ou plutôt skołczować avec un entretien désagréable et non avec un travail en vue de son développement.]

agatalimanowka.pl

Comme c'est habituellement le cas en polonais, le verbe s'accompagne aussi du nom d'action kołczowanie (ou coachowanie) désignant le processus indiqué par celui-là. Du point de vue aspectuel (aspect inaccompli) le mot est équivalent à coaching. Ce qui les distingue cependant c'est la valeur stylistique : la forme polonisée semble être marquée comme ludique et non standard. 
10. Kotczowanie przez gadanie, czyli elementy coachingu w komunikacji.

[Le coaching par la discussion, c'est-à-dire les éléments du coaching dans la communication]

http://wns.sggw.pl

Le même nom d'action, mais précédé du préfixe $s$-, skołczowanie, présente un aspect accompli, comme on peut le constater dans l'exemple suivant:

11. Słowo skołczowanie (...) może (...) kryćzarówno wygłoszenie surowej reprymen$d y$, jak i mobilizujaca rozmowe.

[Le mot ${ }^{*}$ s-coaching (...) peut (...) en cacher autant une réprimande sévère qu'un entretien mobilisant.]

http://coaching.focus.pl/zycie/skolczowani-148

Parmi les dérivés du verbe, notons encore l'adjectif kołczowany, qui peut être aussi un substantif. En désignant celui qui subit l'action en question, il désigne son bénéficiaire, ce que montre la définition suivante:

12. Kołczować oznacza tyle co trenować motywować i rozwijać jednocześnie Przez kołczowanych odbierane to jest jako gnębienie i znęcanie się.

[Kołczować veut dire entraîner, motiver et développer en même temps. Des coachés le conçoivent comme un harcèlement et une persécution.]

http://www.ponglish.org/

Ladjectif correspondant préfixé skołczowany (ou sa variante orthographique scoachowany) présentel'aspect accompli, en désignant celui « qui est déjà coaché»:

13. Już po kilku minutach widać kto jest dobrze scoaczowany (...), mówi Migalski.

[Après quelques minutes déjà on voit qui est bien coaché (...), indique Migalski.] natemat.pl

Cette brève présentation de différents dérivés construits sur la base du mot coach montre qu'il s'agit d'une créativité riche et vivante, compte tenu des changements de sens et de formes des mots construits. Elle s'inscrit essentiellement dans le cadre du système verbal polonais, basé sur l'opposition entre les aspects accompli et inaccompli tout en en développant les noms d'action correspondants, ainsi que les adjectifs déverbaux.

Quant au mot coaching, apparaissant en polonais aussi sous les formes de koaching et kołczing, il ne se soumet pas aussi facilement que coach à la dérivation, probablement en raison de sa terminaison anglaise -ing. En effet, présente 
dans plusieurs autres emprunts en polonais, reconnus comme des noms d'action (comme jogging, trening, timing, dressing), elle bloque la création de dérivés verbaux. Les dérivés adjectivaux semblent cependant possibles et on note la forme coachingowy et kolczingowy (plus rarement) comme adjectif relationnel. Dans l'exemple (14) il accompagne le nom session dont il restreint le champ d'application; il en va de même pour l'exemple (15) dans lequel l'adjectif en question décrit de la même manière le mot programme.

14. Najłatwiej dowiedzieć się jak wygląda sesja coachingowa po prostu z praktyki. Wielu coachów oferuje sesje „demo" podczas pierwszego, darmowego spotkania... [C'est par la pratique qu'on peut apprendre le plus facilement comment se présente une session de coaching. Beaucoup de coachs offrent une session «démo » pendant le premier RV]

swiatcoachingu.pl

\section{Inny modny dziś program kołczingowy dla singielek zaleca: (...)}

[Un autre programme de coaching destiné à des femmes solitaires qui est à la mode aujourd'hui leur indique de : (...). www.facebook.com/psychoterapia.psychoedukacja

Par contre, aussi bien coaching que coach apparaissent comme éléments de mots composés, même si ce dernier est plus rare dans ce cas. Il semble que la série paradigmatique de composés avec coaching ait suivi le modèle du terme anglais life coaching qui s'est implanté en polonais avec son sens d'origine dès les années 2000. Le phénomène désigné par ce terme s’est répandu en Pologne au point de constituer actuellement une spécialisation d'études universitaires, notamment à l'Université de Łódź qui en fait la publicité :

16. Studia podyplomowe life coachingu na Uniwersytecie Łódzkim (...)

[Études post-diplôme du life coaching à l'Université de Łódź (...)]

www.life-coaching.edu.pl

Il est accompagné par le terme life coach (17) qui est cependant moins fréquent.

17. Nazywam się M. O. i jestem life coachem. Oferuję: Sesje indywidualne. W gabinecie w Krakowie orazprzez Skype.

[Je m'appelle M. O. Je suis un life coach. Je vous propose des sessions individuelles. Dans le cabinet à Cracovie ou bien par Skype.]

http://monikaolender.pl/ 
Notons aussi la présence bien établie en polonais d'autres emprunts à l'anglais construits sur le même modèle, notamment skin coaching et skin coach, ainsi que sex coaching et sex coach.

18. Skin coaching zachęca do zmiany myślenia o swojej skórze.

[Le skin coaching t'incite à changer la façon dont tu penses à ta peau.]

www.znak.com.pl

19. Moje klientki często zastanawiają się, czym właściwie różni się skin coach od kosmetyczki. Czy to nie jest tylko chwyt marketingowy?

[Mes clientes se posent souvent la question sur la différence entre le skin coach et l'esthéticienne. N'est-ce pas un truc de marketing ?]

skincoach.pl

20. Sex coaching to proces interdyscyplinarny, bazujacy na terapii poznawczobehawioralnej.

[Le sex coaching est un processus interdisciplinaire, basé sur une thérapie comportementale et cognitive.]

https://niedzwiecka.net

21. W przeciwieństwie do seksuologa, sex coach nie zajmuje się zaburzeniami fizycznymi ani analiza psychologiczna.

[À l'opposé d'un sexologue, le sex coach ne s'occupe pas de troubles physiques ni d'analyse psychologique.]

http://kobiecosc.info/sex-coaching-czym-polega/

Cependant plusieurs composés polonais avec coaching ont un caractère hybride, puisqu' ils contiennent outre l'élément anglais coaching un élément polonais, comme dans le cas du terme coaching zdrowie " coaching santé » (22), bâti sur le modèle $\mathrm{N}+\mathrm{N}$, ou encore coaching jezykowy « coaching de langue » (23), construit sur le modèle $\mathrm{N}+\mathrm{Adj}$.

22. Proponujemy indywidualne konsultacje, coaching zdrowie i kursy doskonalnia. [Nous proposons des consultations individuelles, coaching santé et cours de perfectionnement.]

http://swiatcoachingu.pl 
23. Coaching językowy potrzebny jest uczniom indywidualnym, zmęczonym przez lata nieskutecznej nauki.

[Le coaching de langue est nécessaire pour des élèves individuels, fatigués par des années d'apprentissage inefficace.]

http://ninedots.pl

Notons dans le cas de coaching zdrowie la forme non fléchie du complément de nom $z$ drowie, qui, selon les règles morpho-syntaxiques du polonais, aurait dû avoir la forme du cas génitif, zdrowia.

Signalons aussi le composé coaching finansowy " coaching des finances " (24), dont le deuxième élément, finansowy, constitue aussi un emprunt en polonais (au français), mais déjà adapté à son système morphologique au moyen du suffixe adjectival -owy. Ainsi le composé formé selon le modèle polonais N+ADJ semble constituer un cas à part, intermédiaire entre un composé hybride et un composé comportant les deux éléments étrangers.

24. Coaching finansowy jest dziedzina dość mało popularna, ale ważna.

[Le coaching des finances est un domaine peu populaire, mais important.]

www.coaching4.pl

\section{Les types de procédés d'emprunt}

La liste de mots construits sur la base de coach et coaching en polonais que nous présentons ci-dessus n'est certainement pas exhaustive, mais sans doute renferme-t-elle des emplois fréquents et significatifs. Leur analyse mène à découvrir qu'une ou deux lexies de base empruntées à la langue source peuvent donner lieu à différents types d'emprunts réalisés dans la même langue cible. En effet, en ayant recours à la typologie des emprunts proposée par Jean-François Sablayrolles (2016), il est possible de constater que coach et coaching constituent de vrais emprunts, dans la mesure où ils sont introduits en polonais avec le référent nouveau pour désigner respectivement « entraîneur personnel » et « entraînement personnel », et aussi dans la mesure où ils ont été empruntés pour désigner des référents possédant déjà leur dénomination en polonais, respectivement « entraîneur sportif ", "entraînement sportif» (pol. trener, trenowanie). Il en va de même des termes composés d'éléments anglais, comme life coaching, life coach, sex coaching, sex coach, skin coaching, skin coach qui sont utilisés en anglais. Par contre, selon la même typologie, les dérivés et les composés de 
coach et coaching constituent de faux emprunts, parmi lesquels on distingue des composés hybrides, comme coaching zdrowie, coaching językowy et des mots créés sur la base d'une lexie empruntée, par exemple kołczować, coachka, skołczowany, etc.

\section{Pour conclure}

Même si l'adaptation en polonais des mots construits à partir de coach et coaching apparaît comme avancée, force est de constater que les formes empruntées ou celles qui sont nouvellement créées sur la base d'emprunts sont loin d'être stabilisées en polonais, ce qui s'exprime surtout par la graphie souvent changeante voire fantaisiste des mots en question. Les modifications phonétiques ne sont pas très avancées, ce qui semble résulter de la ressemblance (même si elle n'est qu'approximative) des sons contenus par les mots empruntés. Il est à noter que, systématiquement, les formes plus fortement "polonisées », surtout en ce qui concerne leur graphie, présentent une valeur non formelle, plaisante et / ou péjorative. La dérivation a lieu facilement ; les formes aspectuelles des verbes, mais aussi des adjectifs et des noms déverbaux semblent (quasi) inévitables en polonais. Signalons enfin que les composés hybrides (qui reproduisent pourtant le modèle anglais), abandonnent l'ordre anglais [Déterminant + Déterminé], comme dans life coaching (qui est aussi habituel en polonais), mais prennent l'ordre [Déterminé + Déterminant], non habituel en polonais, comme dans coaching językowy « coaching langagier », peut-être par un besoin inconscient du locuteur de donner ainsi un air « étranger» au mot créé.

\section{Références bibliographiques}

Bańko Mirosław, Czeszewski Maciej, Burzyński Jan, Najnowsze słownictwo polskie, Obserwatorium Językowe UW, http://www.nowewyrazy.uw.edu.pl/. Humbley John, 2016, «La classification des faux emprunts : une question de point de vue ", dans Emprunts néologiques et équivalents autochtones en français, en polonais et en tchèque, Zuzana Hildenbrand, Alicja Kacprzak et Jean-François Sablayrolles (éds.), Limoges, Lambert-Lucas, coll. « La Lexicothèque $»$, p. 36-58.

Humbley John, 2008, «Emprunts, vrais et faux, dans le Petit Robert 2007 », dans Jean Pruvost, Dictionnaires et mots voyageurs. Les 40 ans du Petit Ro- 
bert, de Paul Robert à Alain Rey, Les journées des dictionnaires de Cergy, Herblay, Éditions des Silves, p. 221-238.

Sablayrolles Jean-François, 2016, «Emprunts et influences d'autres langues », dans Emprunts néologiques et équivalents autochtones en français, en polonais et en tchèque, Zuzana Hildenbrand, Alicja Kacprzak et Jean-François Sablayrolles (éds.), Limoges, coll. « La Lexicothèque », Lambert-Lucas, p. 23-35.

Sablayrolles Jean-François, 2000, La néologie en français contemporain, Examen du concept et analyse de productions néologiques récentes, Paris, Honoré Champion.

Winter-Froemel Esme, 2009, «Les emprunts linguistiques : enjeux théoriques et perspectives nouvelles», Neologica, 3, p. 79-122. 